\title{
ARTICLE
}

\section{Influence of the patient size on secondary doses delivered in light ion beams}

\author{
Irena Gudowska* and Martha Hultqvist \\ Medical Radiation Physics, Department of Physics, Stockholm University, Box 260, 17176 Stockholm, Sweden
}

\begin{abstract}
Secondary radiation exposure of patients in light ion therapy is of concern due to possible normal tissue damage and risk of induction of secondary cancers. Neutrons, protons and heavier ions are generated by nuclear inelastic interactions of primary ions both in the beam line and in the patient. The patient is exposed to a complex radiation field and secondary doses can be deposited in normal tissues both close to and relatively far from the treated volume. The energy distribution of secondary particles and secondary doses delivered to different organs were studied by the MC code SHIELD-HIT10 using the anthropomorphic phantoms representing a 10-year-old child (CHILD-HIT) and an adult male (ADAM-HIT). Brain tumor irradiations were simulated with approximated scanned beams of ${ }^{1} \mathrm{H},{ }^{7} \mathrm{Li}$ and ${ }^{12} \mathrm{C}$ ions. The influence of patient size on the secondary dose distributions were studied using the same target definition and irradiation geometry with a lateral beam. For the scanned beams, the secondary organ absorbed doses normalized per absorbed dose to the treated volume (brain tumor) were in the range $1 \mathrm{nGy} / \mathrm{Gy}-0.1 \mathrm{mGy} / \mathrm{Gy}$ and the absorbed doses in the CHILD-HIT phantom were higher than in ADAM-HIT by up to a factor of 5, depending on organ.
\end{abstract}

Keywords: secondary doses; light ion therapy; Monte Carlo simulations

\section{Introduction}

The exposure of normal tissues to radiation during radiotherapy raises the concern of the induction of side effects. With the increasing success rate of radiotherapy and therefore an increasing number of long-term survivors (especially true for paediatric patients) the induction of secondary cancers is a matter of concern [1-4].

In light ion therapy with ions in the energy range 100 - $400 \mathrm{MeV} / \mathrm{u}$, the complex radiation field of secondary particles is generated due to nuclear inelastic reactions with the beam-line components and the patient. The produced secondary radiation includes neutrons, protons and heavier ions of energies from a few $\mathrm{keV} / \mathrm{u}$ up to 1 $\mathrm{GeV} / \mathrm{u}$ characterized by the wide ranges of LET. The secondary particles themselves generate secondaries in a cascade of events during their transport through the patient and deliver dose to the out-of-field organs.

Monte Carlo simulations coupled with anthropomorphic phantoms in order to calculate secondary organ doses have mostly been performed for proton therapy [5-7]. A few studies of simulated secondary doses have been published for ions heavier than protons [8-10]. The secondary organ absorbed doses simulated for approximated scanned beams (no beam line components) were low and on the order of $10^{-6}-10^{-1}$ mGy per Gy to the target, with a general

*Corresponding author. Email: irena.gudowska@ki.se decrease with increasing distance from the target volume.

Studies of secondary doses in proton therapy for brain lesions as a function of phantom age have previously been performed by Zacharatou-Jarlskog and coworkers [7] who simulated organ doses with the GEANT4 code coupled with whole-body age-dependent voxel phantoms. They recognized that the increasing distance between organs and target volume with increasing phantom size resulted in decreasing neutron organ equivalent doses.

The aim of the present studies was to investigate the influence of patient size on the secondary dose distributions for ions heavier than protons. The results of the secondary dose are reported as absorbed doses. Equivalent doses and risk estimation are not discussed here due to large uncertainties in the radiation weighting factors $w_{R}$ of the secondary radiation and very low secondary absorbed doses encountered in ion therapy.

\section{Materials and method}

In order to investigate the influence of patient size on the secondary doses delivered in light ion beams, simulations were performed with the Monte Carlo (MC) code SHIELD-HIT10 [11] coupled with anthropomorphic phantoms representing an adult male (ADAM-HIT) and a 10-year-old child (CHILD-HIT). These phantoms were constructed on the basis of the 
Oak Ridge National Laboratory Mathematical anthropomorphic phantoms $[12,13]$ to comply with the geometrical description in SHIELD-HIT10 [10].

MC simulations with ADAM-HIT and CHILD-HIT were performed using the same target definition and irradiation geometry. Simulations with primary ion beams of ${ }^{1} \mathrm{H},{ }^{7} \mathrm{Li}$ and ${ }^{12} \mathrm{C}$ ions in the energy range $110-$ $212 \mathrm{MeV} / \mathrm{u}$ were performed for brain tumour irradiation.

The anthropomorphic phantoms ADAM-HIT and CHILD-HIT are illustrated in Figure 1. The tumor was in both phantoms modeled as a cylinder of $3 \mathrm{~cm}$ length and $3 \mathrm{~cm}$ diameter located in the center of the brain. The irradiations were performed with one left lateral circular mono-energetic ion beam with diameter $2.8 \mathrm{~cm}$, which resulted in non-uniform dose distribution delivered the tumor. Since the head of CHILD-HIT (distal end of tumor was located at $8.9 \mathrm{~cm}$ depth) was smaller than that of ADAM-HIT (distal end of tumor at $9.5 \mathrm{~cm}$ depth), slightly higher energies of the primary ions were required for ADAM-HIT. The fact that the spread out Bragg peak (SOBP) was not modeled in these studies does not result in significant differences in the doses absorbed by organs further away from the tumor volume as it was shown by Hultqvist and Gudowska [10]. The organ absorbed doses for secondary charged fragments were calculated in SHIELD-HIT10 as energy deposited to the organ divided by the organ mass. In order to evaluate the neutron absorbed organ doses, two sets of simulations were performed for each studied beam: one simulation with all particles generated and transported in the hadron cascade, and one simulation with all particles generated but with no further transport of the produced neutrons. The differences in the absorbed doses obtained in these two simulations give an estimation of the dose contribution from the produced neutrons.

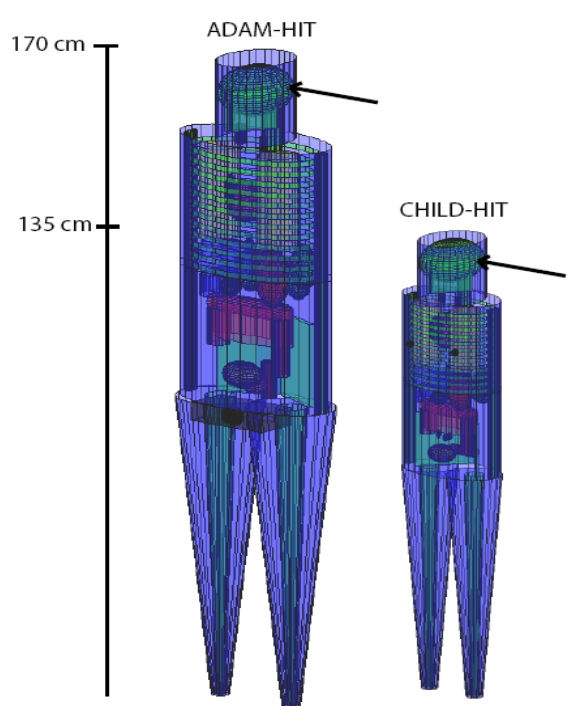

Figure 1. The anthropomorphic phantoms used in simulations with the Monte Carlo code SHIELD-HIT; for male - ADAM-HIT and 10 years old child - CHILD-HIT. The geometry of brain tumor irradiation with one left lateral beam is shown.

\section{Results and discussion}

Secondary particle fluences differential in energy in the thyroid generated under brain tumor irradiation with ${ }^{12} \mathrm{C}$ beams, are presented in Figure 2 and Figure 3 for CHILD-HIT and ADAM-HIT, respectively.

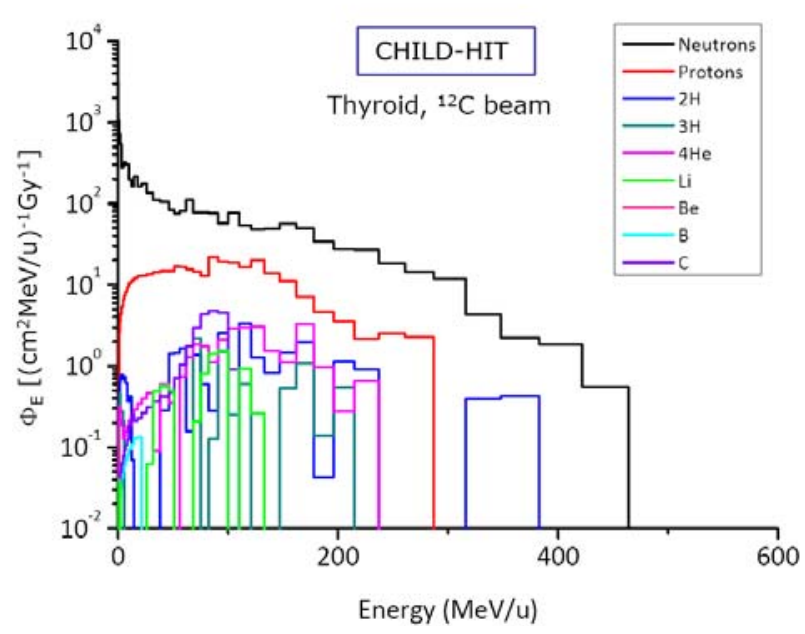

Figure 2. Secondary particle fluences differential in energy normalized to the target absorbed dose in the thyroid of CHILD-HIT. Brain tumor irradiation with ${ }^{12} \mathrm{C}$ beams of 205 $\mathrm{MeV} / \mathrm{u}$.

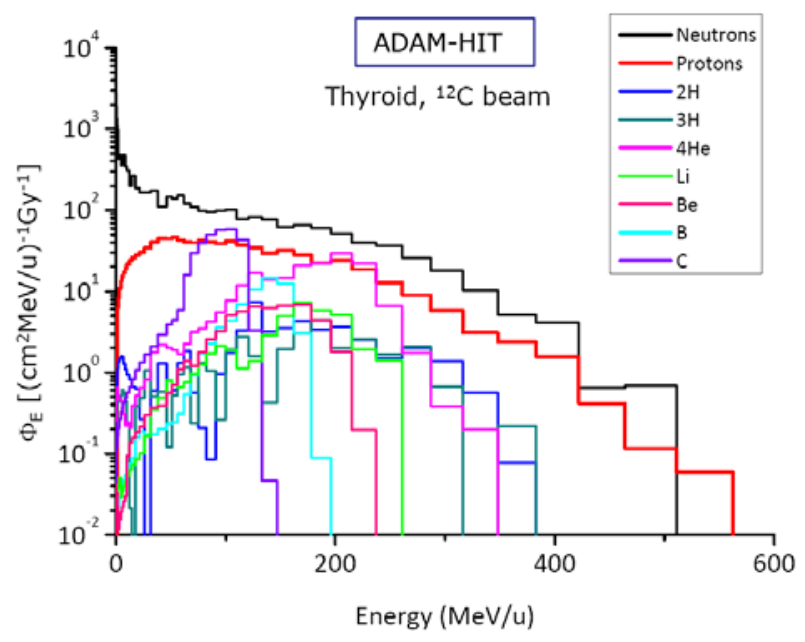

Figure 3. Secondary particle fluences differential in energy normalized to the target absorbed dose in the thyroid of ADAM-HIT. Brain tumor irradiation with ${ }^{12} \mathrm{C}$ beams of 212 $\mathrm{MeV} / \mathrm{u}$.

The fluences and maximum energies of secondary neutrons, protons, deuterium, helium and heavier fragments are generally larger in the thyroid of ADAM-HIT compared to CHILD-HIT. Particle fluences were averaged over larger thyroid volume of ADAM-HIT and more nuclear interactions is expected in the male phantom. On the other hand high energy secondary particles are easily transported outside the smaller CHILD-HIT phantom.

The evaluated secondary organ absorbed doses 
normalized to target (brain tumour) absorbed dose in CHILD-HIT and ADAM-HIT were in the range 1 nGy/Gy - 0.1 mGy/Gy.

Figure 4 show organ absorbed doses in thyroid, lung, heart, stomach and bladder of CHILD-HIT for irradiation with ${ }^{1} \mathrm{H}(110 \mathrm{MeV}),{ }^{7} \mathrm{Li}(126 \mathrm{MeV} / \mathrm{u})$ and ${ }^{12} \mathrm{C}$ $(205 \mathrm{MeV} / \mathrm{u})$ primary ions. Figure 5 presents the dose distributions for ADAM-HIT irradiated with ${ }^{1} \mathrm{H}$ (113 $\mathrm{MeV}),{ }^{7} \mathrm{Li}(130 \mathrm{MeV} / \mathrm{u})$ and ${ }^{12} \mathrm{C}(212 \mathrm{MeV} / \mathrm{u})$ primary ions.

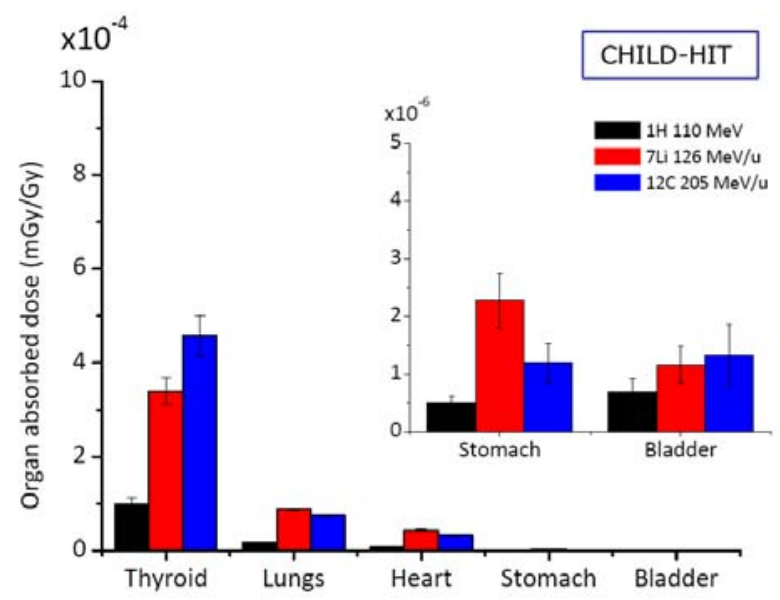

Figure 4. Organ absorbed doses per absorbed dose to target in CHILD-HIT for irradiation with ${ }^{1} \mathrm{H},{ }^{7} \mathrm{Li}$ and ${ }^{12} \mathrm{C}$ primary ions. The error bars show one standard deviation in the calculated absorbed organ doses.

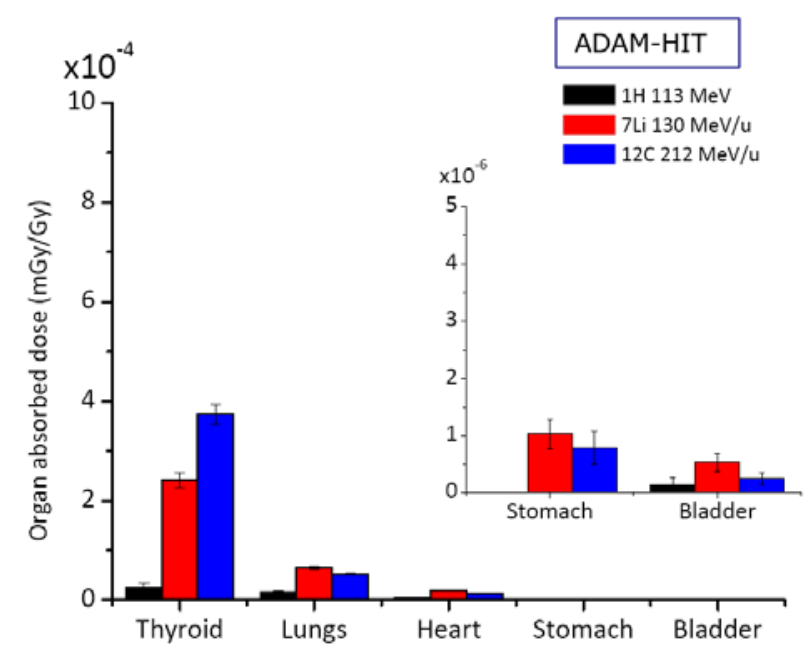

Figure 5. Organ absorbed doses per absorbed dose to target in ADAM-HIT for irradiation with ${ }^{1} \mathrm{H},{ }^{7} \mathrm{Li}$ and ${ }^{12} \mathrm{C}$ primary ions. The error bars show one standard deviation in the calculated absorbed organ doses.

In general, lower organ absorbed doses in the adult phantom are observed since the organs are located at larger distances from the target volume and the size of the organs is larger compared to the child phantom. For organs like stomach and bladder the absorbed doses in the CHILD-HIT were higher than in ADAM-HIT by up to a factor of 5 .

The dose absorbed by the brain (excluding the tumor volume and the volume irradiated by the primary beam), not shown in Figure 4, was $0.05,0.09$ and $0.1 \mathrm{mGy} / \mathrm{Gy}$ in CHILD-HIT for the ${ }^{1} \mathrm{H},{ }^{7} \mathrm{Li}$ and ${ }^{12} \mathrm{C}$ beams, respectively.

The differences in absorbed brain doses in ADAM-HIT and CHILD-HIT were smaller than for the other organs: the brain absorbed doses in CHILD-HIT and ADAM-HIT differed by $10 \%$ in the ${ }^{1} \mathrm{H}$ beam, while the difference was less than $1 \%$ in the ${ }^{7} \mathrm{Li}$ and ${ }^{12} \mathrm{C}$ beam.

As it was also observed and pointed out by Zacharatou-Jarlskog et al. [7], the distance from the target volume remains largely unchanged in organs located very close to the target volume and the influence of the patient size on the absorbed dose to these organs therefore decreases.

The separate dose contributions from charged fragments and neutrons to the secondary organ absorbed doses in ADAM-HIT and CHILD-HIT were evaluated. The secondary neutrons were the sole contributor to the organ absorbed doses in the simulated ${ }^{1} \mathrm{H}$ beam. About $17 \%$ and $58 \%$ of the dose absorbed by the thyroid in CHILD-HIT was due to charged fragments in the simulated ${ }^{7} \mathrm{Li}$ and ${ }^{12} \mathrm{C}$ beams, respectively. The corresponding numbers for the thyroid in ADAM-HIT were $14 \%$ and $47 \%$. The contribution of charged fragments to the absorbed dose to the lungs was about $1 \%$ in both phantoms in the simulated ${ }^{7} \mathrm{Li}$ beam, while the contributions in the ${ }^{12} \mathrm{C}$ beams were $18 \%$ in CHILD-HIT and $17 \%$ in ADAM-HIT. A contribution to the heart absorbed dose from charged fragments was only obtained in the ${ }^{12} \mathrm{C}$ beam, and on the level of $8 \%$ in CHILD-HIT and 2\% in ADAM-HIT. Also a decrease in the charged fragment contribution with increasing distance from the target volume is observed. Since the organs in ADAM-HIT were located at larger distances from the target volume than in CHILD-HIT, the dose contribution from charged fragments was in general lower in ADAM-HIT than in CHILD-HIT.

\section{Conclusion}

Secondary out-of-field absorbed doses in light ion therapy were evaluated in the human anthropomorphic phantoms of different sizes. The MC code SHIELD-HIT10 coupled with the anthropomorphic phantoms representing a 10-year-old child (CHILD-HIT) and an adult male (ADAM-HIT) was used in these studies. Brain tumor irradiation with ${ }^{1} \mathrm{H}$, ${ }^{7} \mathrm{Li}$ and ${ }^{12} \mathrm{C}$ ions in the energy range $110-212 \mathrm{MeV} / \mathrm{u}$ was simulated using the same target definition and irradiation geometry.

The results of secondary particle fluences show that a complex secondary radiation field can be delivered to normal tissues both close to and relatively far from the treated volume.

For the scanned beams, the secondary organ absorbed 
doses normalized per absorbed dose to the treated volume (brain tumor) were in the range $1 \mathrm{nGy} / \mathrm{Gy}-0.1$ $\mathrm{mGy} / \mathrm{Gy}$. Since the distances between organs and the treated volume are generally smaller in a child than in an adult, larger radiation doses are delivered to the organs of a paediatric patient than of an adult patient.

The absorbed doses in the CHILD-HIT phantom were higher than in ADAM-HIT by up to a factor of 5 , depending on organ size. Secondary organ doses are highly dependent on the irradiation geometry, size of the human phantom, and type and energy of the primary ion beam.

The secondary neutrons were the sole contributor to the organ absorbed doses in the simulated ${ }^{1} \mathrm{H}$ beam. For the beams of ions heavier than ${ }^{1} \mathrm{H}$, the dose contributions from charged fragments which were not products of neutron interaction was observed not only in organs located in the direct vicinity of the target volume, but also in organs located far from the treated volume. The dose contribution from charged fragments was in general lower in ADAM-HIT than in CHILD-HIT.

\section{Acknowledgements}

The work was partially supported by Swedish Radiation Safety Authority under the contract SSM2012-1998.

\section{References}

[1] E.J. Hall and C.S. Wuu, Radiation-induced second cancers: The impact of 3D-CRT and IMRT, Int. J. Radiat. Oncol. Biol. Phys. 56 (2003), pp. 83-88.

[2] S.F. Kry, M. Salehpour, D.S. Followill, M. Stovall, D.A. Kuban, A. White and I.I. Rose, The calculated risk of fatal secondary malignancies from intensity-modulated radiation therapy, Int. J. Radiat. Oncol. Biol. Phys. 62 (2005), pp. $1195-1203$.

[3] G.X. Xu, B. Bednarz and H. Paganetti, A review of dosimetry studies on external-beam radiation treatment with respect to second cancer induction, Phys. Med. Biol. 53 (2008), R191-R241.
[4] W.D. Newhauser and M. Durante, Assessing the risk of second malignancies after modern Radiotherapy, Nat. Rev. Cancer 11 (2011), pp. 438-448.

[5] H. Jiang, B. Wang, X.G. Xu, H.D. Suit and H. Paganetti, Simulation of organ-specific patient effective dose due to secondary neutrons in proton radiation treatment, Phys. Med. Biol. 50 (2005), pp. 4337-4353.

[6] P.J. Taddei, D. Mirkovic, J.D. Fontenot, A. Giebeler, Y. Zheng, D. Kornguth, R. Mohan and W. Newhauser, Stray radiation dose and second cancer risk for a pediatric patient receiving craniospinal irradiation with proton beams, Phys. Med. Biol. 54 (2009), pp. 2259-2275.

[7] C. Zacharatou Jarlskog, C. Lee, W. Bolch, X.G. Xu and H. Paganetti, Assessment of organ specific neutron doses in proton therapy using whole-body age-dependent voxel phantoms, Phys. Med. Biol. 53 (2008), pp. 693-717.

[8] T. Sato, Y. Kase, R. Watanabe, K. Niita and L. Sihver, Biological dose estimation for charged-particle therapy using an improved PHITS code coupled with a microdosimetric kinetic model, Radiat. Res. 171 (2009), pp. 107-117.

[9] M. Hultqvist and I. Gudowska, Secondary doses delivered to an anthropomorphic male phantom under prostate irradiation with proton and carbon ion beams, Radiat.Meas. 45 (2010), pp. 1410-1413.

[10] M. Hultqvist and I. Gudowska, Secondary absorbed doses from light ion irradiation in anthropomorphic phantoms representing an adult male and a 10 year old child, Phys. Med. Biol. 55 (2010), pp. 6633-6653.

[11] Sobolevsky $2010 \mathrm{http}: / / \mathrm{www} . \mathrm{inr}$. ru/shield

[12] M. Cristy and K.F. Eckerman, Specific Absorbed Fractions of Energy At Various Ages from Internal Photon Sources, ORNL/TM-8381 Oak Ridge, TN: Oak Ridge National Laboratory, (1987)

[13] ORNL 2009 Description of the mathematical phantoms, http://ordose.ornl.gov/resources/phantom.html 